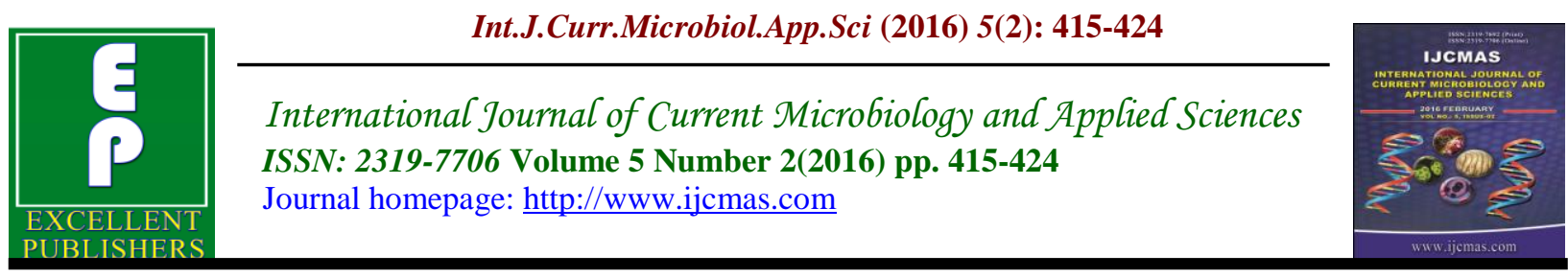

Original Research Article

doi: http://dx.doi.org/10.20546/ijcmas.2016.502.046

\title{
Study of Biofilm Production and Anti-microbial susceptibility pattern of bacterial and fungal isolates from urinary catheters
}

\author{
Venkata Hemalatha Neeli, T. Parvathi* and Perala Balamurali Krishna \\ Department of Microbiology, Andhra Medical College, Visakhapatnam, India \\ *Corresponding author
}

\begin{abstract}
A B S T R A C T
Keywords

Biofilm,

Urinary catheter

tips,

Tube Method,

Congo Red Agar

method,

Tissue Culture

Plate method

Article Info

Accepted:

22 January 2016

Available Online:

10, February 2016

A biofilm is an accumulation of microorganisms embedded in an exopolysaccharide matrix of microbial and host origin called Polysaccharide Intercellular Adhesin (PIA). They pose a great threat to indwelling devices causing slow, persistent infections; thereby interfering with antibiotic therapy. This study was carried out to isolate and identify the bacteria and fungi from the tips of urinary catheters, to determine their anti-microbial susceptibility patterns and to detect their ability to produce biofilm by three different methods. 132 urinary catheter tips were collected from patients admitted into various wards and the organisms isolated were subjected to three different tests to detect their ability to produce biofilms, i.e., a) Tube Method, b) Congo Red Agar method and c) Tissue Culture Plate method. 49 samples showed culture positivity; out of which, 18 produced biofilms. TCP method detected $42 \%$ biofilm producers whereas Tube method and Congo red agar method detected $32 \%$ and $26 \%$ respectively. Antimicrobial susceptibility testing was done and increased resistance was shown by biofilm producers. The information on the capacity of a clinical isolate to produce biofilm would help a clinician to evaluate the measure of its virulence and devise an appropriate treatment plan for the patient.
\end{abstract}

\section{Introduction}

A biofilm is an accumulation of microorganisms embedded in an exopolysaccharide matrix of microbial and host origin called Polysaccharide Intercellular Adhesin (PIA) [2,7,11,14]. Microorganisms living in a biofilm are capable of adheringirreversibly to each other or to other surfaces ${ }^{[2,14]}$. Thus, a spatially organized three-dimensional structure is produced ${ }^{[2]}$. They exhibit an altered phenotype with respect to growth rate and gene transcription [2]. Within a biofilm, bacteria communicate with each other by the production of chemical signals or inducer molecules, a phenomenon called 'quorum sensing' ${ }^{[2]}$. Examples include chemical signaling through acyl homoserine lactone (AHL) in Gram-negative bacteria and oligopeptides in Gram-positive bacteria ${ }^{[14]}$. Availability of key nutrients, chemotaxis towards the 
surface, motility of bacteria, presence of surface adhesins and surfactants are some of the key factors influencingbiofilm formation [2]

Micro-organisms growing in a biofilm are intrinsically more resistant to anti-microbial agents than their planktonic (free-floating) counterparts. Interestingly, antibiotic resistance can increase a ' 1000 fold' ${ }^{[2]}$. This is because of the failure of the antibiotic to penetrate the polysaccharide matrix. In addition, some of the cells in the biofilm may experience nutrition deprivation and therefore exist in a slow-growing or starved state, displaying reduced susceptibility to antimicrobial agents ${ }^{[14]}$. Therefore, higher anti-microbial concentrations are required to inactivate the organisms growing in a biofilm ${ }^{[2]}$. The proximity of cells within a biofilm can facilitate exchange of plasmids responsible for drug resistance and hence enhance the spread of anti-microbial resistance ${ }^{[13]}$. Biofilms impede penetration not only of antibiotics but also of variety of disinfectants, which emphasizes that their characterization is important aspect of 'infection control ${ }^{,[2,10,11]}$. Biofilms thus form a major virulence factor of bacteria and are considered as markers of clinically relevant infection ${ }^{[8,10]}$

Normally, the urinary tract is protected from microbial colonization by the flushing action of sterile urine, the sloughing of uroepithelial cells and glycosaminoglycan layer [4]. When an indwelling catheter is left in place, the bacteria capable of producing biofilms attach to the surface of catheter and play a major role in decreasing the susceptibility to anti-microbial agents [13]. Catheters are initially colonized by single species of micro-organisms - monomicrobic aggregation. As the catheter remains inplace for a longer duration, the number and diversity of organisms increase - polymicrobic aggregation ${ }^{[9,14]}$. In this way, biofilms cause chronic infections which are difficult to treat and lead to longer hospitalization time resulting in much higher treatment costs ${ }^{[13]}$.

The biofilm-forming communities are able to adapt and respond to changes in their environment, similar to a multicellular organism. A variety of bacterial pathogens, along with other microorganisms, are capable of forming biofilms, including Gram positive cocci, Gram negative bacilli and Candida albicans ${ }^{[14]}$.

The aim and objectives of this study includes; to isolate and identify the bacteria and fungi from the tips of urinary catheters. To determine their anti-microbial susceptibility patterns. And to detect their ability to produce biofilm by three different methods, i.e., Tube Method (TM), Congo Red Agar (CRA) method and Tissue Culture Plate (TCP) method.

\section{Materials and Methods}

The present study was conducted at King George Hospital (KGH), Visakhapatnam over a period of six months, i.e., from May 2015 to October 2015. Under strict aseptic conditions, a total of 132 urinary catheter tips were collected into sterile universal containers (Figure 1) from patients admitted into gynaecology and medicine wards and were processed.

Organisms were initially isolated on routine culture media like nutrient agar, blood agar and MacConkey agar and were identified by standard microbiological procedures, i.e., by cultural characteristics, Gram's stain, catalase test, oxidase test, motility and other biochemical tests. Antimicrobial susceptibility testing was done by modified Kirby Bauer disc diffusion method on 
Mueller Hinton agar. The organismsthus identified were subjected to three different tests to detect their ability to produce biofilms.

\section{Biofilm Detection Methods}

\section{Tube Method (TM)}

This qualitative method for the assessment of biofilm formation was described by Christensen et al ${ }^{[2]}$.

Five milliliters of trypticase soy broth (TSB)with $1 \%$ glucose was inoculated with a loopful of the test organism from overnight culture plates. Inoculated broths were incubated at $37^{\circ} \mathrm{C}$ for 48 hours. The contents were gently decanted, washed with phosphate buffer saline $(\mathrm{pH} 7.3)$ and left to dry at room temperature. The tubes were then stained with $0.1 \%$ safranine. Each tube was gently rotated to ensure uniform staining and after 1 minute, its contents were decanted. They were dried in inverted position and observed for biofilm formation.

Biofilm formation was considered positive when a visible film lined the wall and bottom of the tube. Ring formation at the liquid interface was not indicative of biofilm formation. Tubes were examined and the results were scored visually as (Figure 2)

0 - Absent, 1 - Weak,2 - Moderate and 3 - Strong.

\section{Congo Red Agar (CRA) Method}

This method, developed by Freeman et al is based on the characteristic cultural morphology of biofilm-forming bacteria on Congo-red agar medium ${ }^{[2]}$. The composition of the medium is BHI broth $37 \mathrm{gm} / \mathrm{L}$; Sucrose $50 \mathrm{gm} / \mathrm{L}$; Agar $10 \mathrm{gm} / \mathrm{L}$; Congo red $0.8 \mathrm{gm} / \mathrm{L}$
Congo red stain was prepared as a concentrated aqueous solution and autoclaved separately from the other media constituents. It was then supplemented to the autoclaved BHI agar with sucrose, when its temperature reached $55^{\circ} \mathrm{C}$. Plates were inoculated with the test organism and incubated overnight at $37^{\circ} \mathrm{C}$.

Black colonies with a dry crystalline consistency indicated strong biofilm production (Figure 3). Weak biofilm producers showed red colonies with occasional darkening at the center (Figure 4) and non-biofilm producers usually remained pink to red (Figure 5).

This property was found to be lost in repeated sub-culture (Figure 6).

\section{Tissue Culture Plate (TCP) Method}

This quantitative test was described by Christensen et $\mathrm{al}^{[2]}$. Overnight growth of bacteria in trypticase soy broth (TSB) with $1 \%$ glucose was diluted 1:100 with fresh medium. Individual wells of 96 welled, sterile, polystyrene microtitreplate were filled with $200 \mu \mathrm{l}$ of diluted cultures. Positive control well contained biofilm producer, Pseudomonas aeruginosa ATCC 27853 strain and negative control well contained sterile TSB.

The plates were incubated at $37^{\circ} \mathrm{C}$ for 24 hours. After incubation, contents of each well were removed by gentle tapping. The wells were washed three times with phosphate buffer saline ( $\mathrm{pH} 7.3$ ) to remove free-floating 'planktonic' bacteria. Biofilm formed by bacteria adherent to the wells was fixed with $2 \%$ sodium acetate and was stained with $0.1 \%$ safranine. Excess stain was removed by through washing with deionized water and plates were air dried (Figure 7). 
Optical density of the stained adherent biofilm was measured at $570 \mathrm{~nm}$ using ELISA reader. The interpretation of the biofilm production was done according to the criteria of Stephanovicet et al.

$\underline{\text { OD valueBiofilm production }}$

$\leq 2 \mathrm{x}$ ODcnon/weak biofilm producer

$2 \mathrm{x}$ ODc to $\leq 4 \mathrm{x}$ ODcmoderate biofilm producer

$\geq 4 x$ ODcstrong biofilm producer

(Optical density cut-off value $(\mathrm{ODc})=\mathrm{OD}$ of negative control $+3 \mathrm{SD}$ of negative control)

\section{Results and Discussion}

In the present study, out of 132 samples processed, 49 were culture positive for both bacterial and fungal isolates (37\%) and 83 were culture sterile (63\%)(Diagram 1).

Out of 49 isolates obtained, 18 showed positive biofilm production. Among them, 18 isolates showed biofilm production by Tissue culture plate method, 14 by Tube method and 11 by Congo red agar method. This finding suggests that TCP method detects more biofilm producers $(42 \%)$ when compared to Tube method (32\%) and CRA method (26\%) (Diagram 2).

The 18 isolates that showed positive biofilm production in the present study were Escherichia coli (8), Klebsiella spp. (6), Pseudomonas sp.(2) and Candida (2) (Diagram 3).

In the present study, among the bacterial isolates, only Gram negative bacteria were isolated and 16 of them produced biofilms. Gram positive bacteria were not isolated.
This might be because of the fact that the samples collected were 'urinary catheter tips' which were free from resident microbiota of the urethra and skin contaminants.

Among the positive biofilm producers, 6 isolates were obtained from catheters which remained in place for about 2 weeks duration. 3 isolates each were obtained from those that remained for 1 week and 10 days and 2 isolates each from catheters of 5 days and 10 days duration. This finding suggests that the chances of biofilm formation increases with the duration of catheterization. Longer the catheter remains in place; more are the chances of biofilm formation (Diagram 4)

Antimicrobial susceptibility testing (AST) was done against Nitrofurantoin $300 \mu \mathrm{g}$, Nalidixic acid $30 \mu \mathrm{g}$,Norfloxacin $10 \mu \mathrm{g}$, Ceftazidime $30 \mu \mathrm{g}$,Amikacin $30 \mu \mathrm{g}$ and Amoxyclav $30 \mu \mathrm{g} /$ disc on Mueller Hinton agar by Modified Kirby Bauer disc diffusion method. Susceptibility was determined after measuring the zones of inhibition and comparing them with that of standard zone size interpretation chart (Table 1).

Antifungal susceptibility testing for Candida was done as per CLSI M44-A2 guidelines on Mueller Hinton agar supplemented with $2 \%$ glucose and methylene blue against

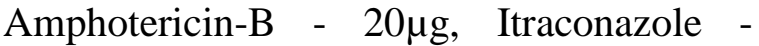

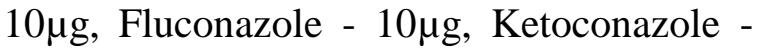
$10 \mu \mathrm{g}$, Clotrimazole - 10 $\mathrm{g}$ and Nystatin 100units/disc (Table 2).

In the present study, Tissue Culture Plate (TCP) method detected more number of isolates $(42 \%)$ capable of producing biofilm when compared to Tube method and Congo red agar (CRA) method. Similar findings were observed by Hassan A, et al., (2011), Anuradha De, et al., (2012). 
Table.1 Anti-microbial Susceptibility Testing for Bacterial Isolates Producing Biofilms

\begin{tabular}{|c|c|c|c|}
\hline & Susceptible & Intermediate & Resistant \\
\hline Nitrofurantoin & $50 \%$ & $12.5 \%$ & $37.5 \%$ \\
\hline Nalidixic acid & $18.75 \%$ & $18.75 \%$ & $62.5 \%$ \\
\hline Norfloxacin & $31.25 \%$ & $25 \%$ & $43.75 \%$ \\
\hline Ceftazidime & $18.75 \%$ & $31.25 \%$ & $50 \%$ \\
\hline Amikacin & $25 \%$ & $18.75 \%$ & $56.25 \%$ \\
\hline Amoxyclav & $25 \%$ & $12.5 \%$ & $62.5 \%$ \\
\hline
\end{tabular}

Table.2 Anti-fungal Susceptibility Testing for Fungal Isolates Producing Biofilms

\begin{tabular}{|c|c|c|c|}
\hline & Susceptible & Intermediate & Resistant \\
\hline Amphotericin-B & $50 \%$ & - & $50 \%$ \\
\hline Itraconazole & - & - & $100 \%$ \\
\hline Fluconazole & $50 \%$ & - & $50 \%$ \\
\hline Ketoconazole & $50 \%$ & - & $50 \%$ \\
\hline Clotrimazole & - & $50 \%$ & $50 \%$ \\
\hline Nystatin & $100 \%$ & - & - \\
\hline
\end{tabular}

Figure.1 Tips of Foley's Catheters in Sterile Universal Containers

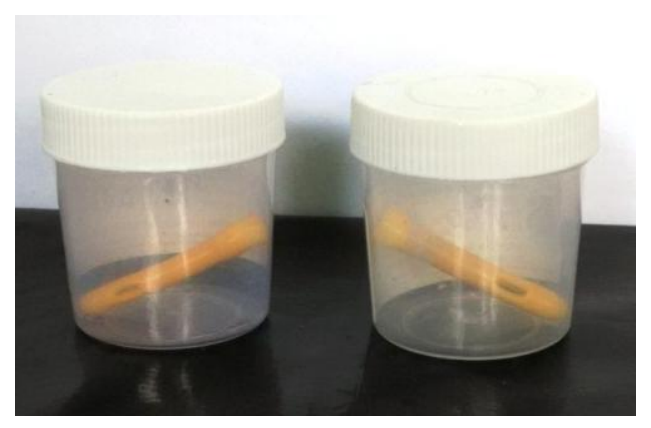

Figure.2 Tube Method for Detection of Biofilm Production

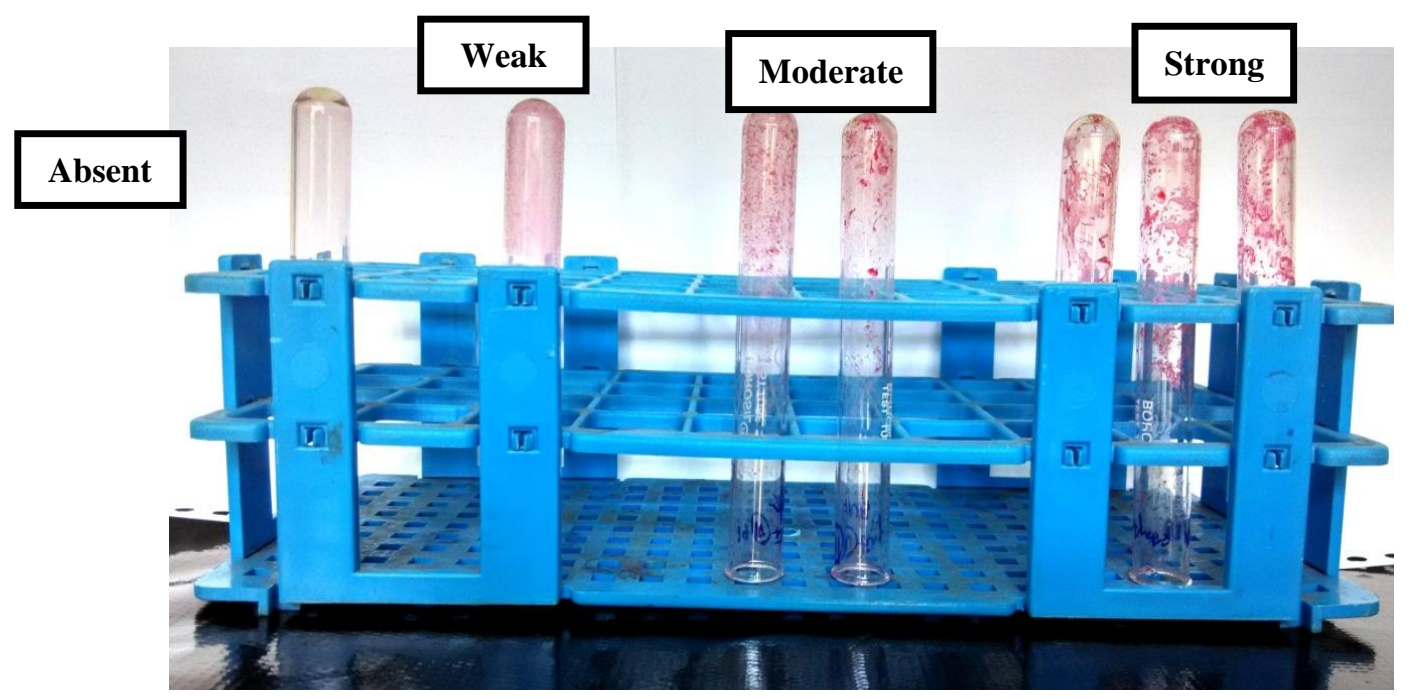


Figure.3 Strong Biofilm Producer on Congo Red Agar

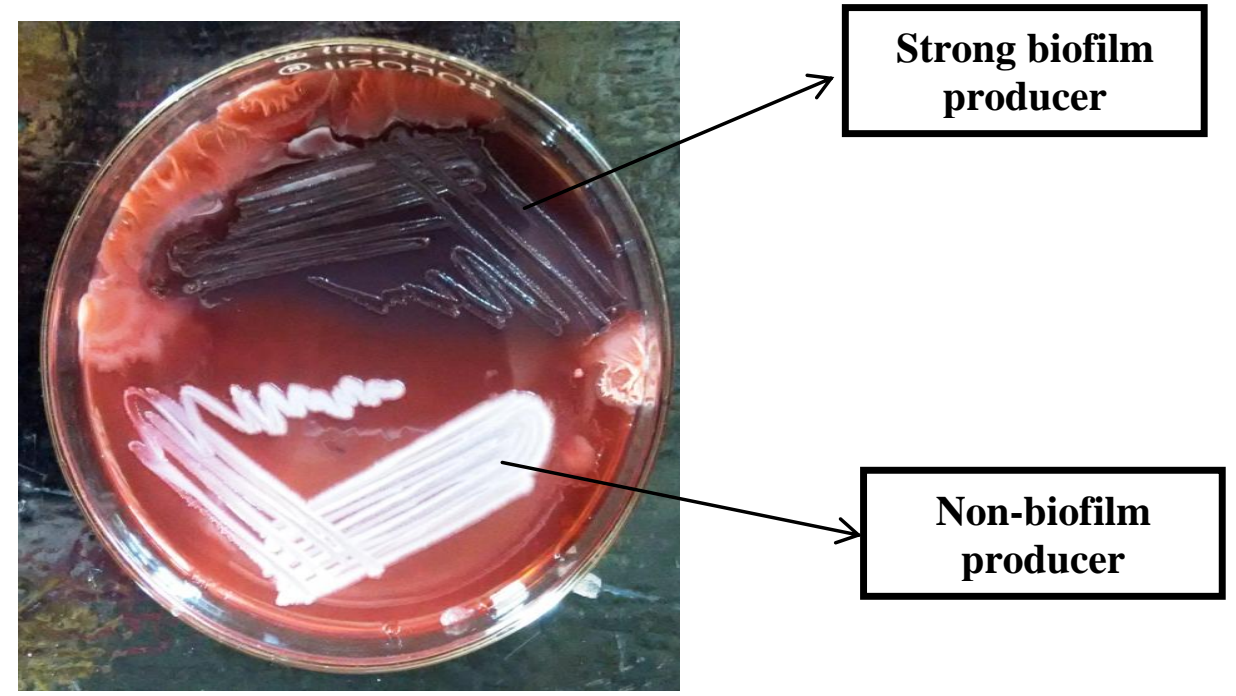

Figure.4 Weak Biofilm Producer on Congo Red Agar

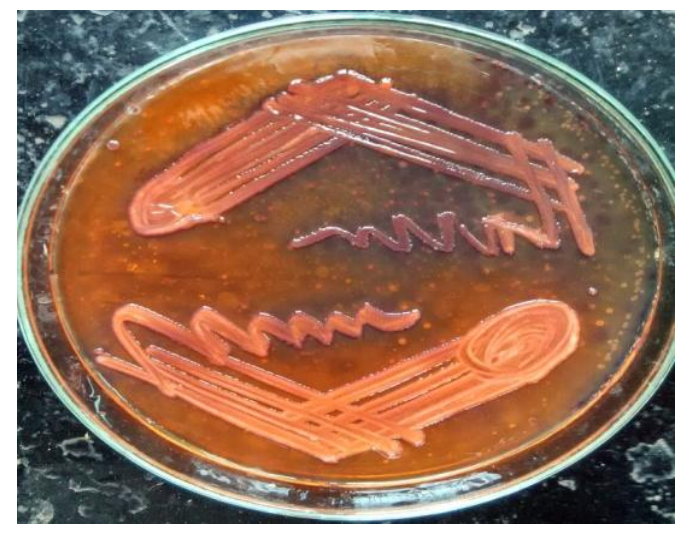

Figure.5 Non Biofilm Producer on Congo Red Agar

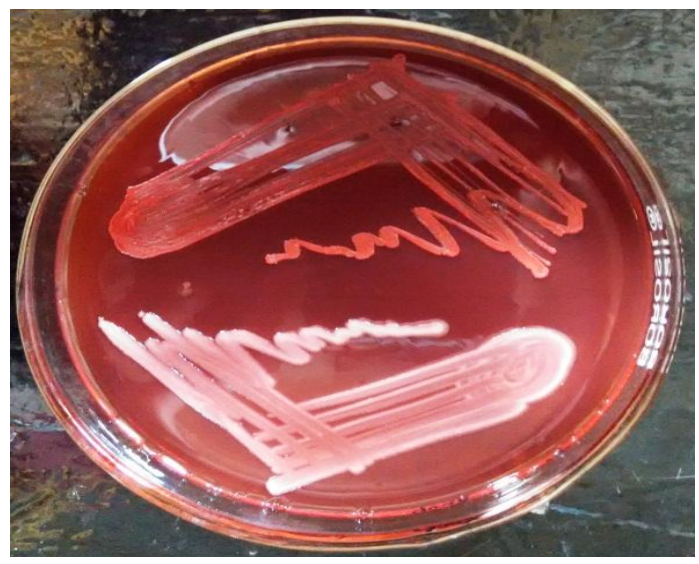


Figure.6 Loss of Biofilm Forming Ability on Sub-culture

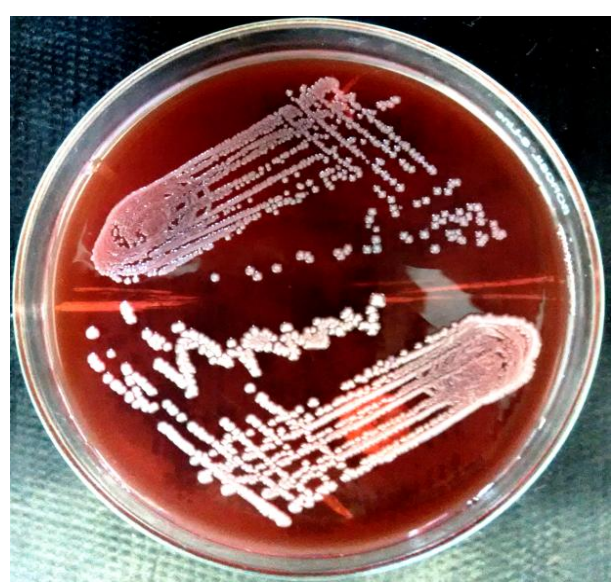

Figure.7 Tissue Culture Plate Method for the Detection of Biofilm Production

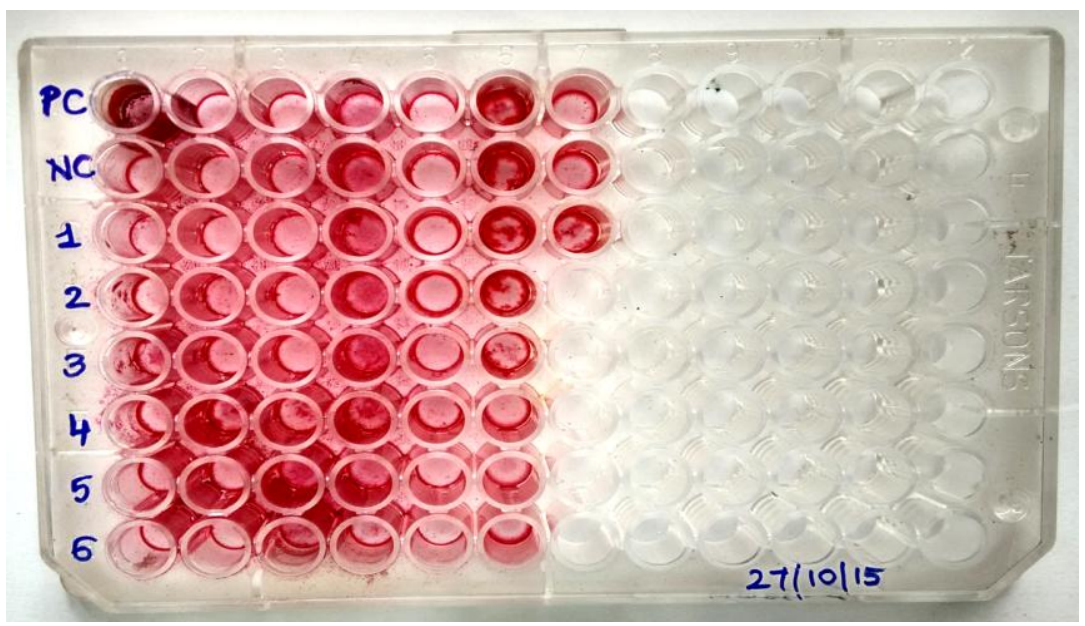

Diagram.1 Pie Diagram Showing Percentage of Culture Positivity

\section{Culture positivity}

Culture sterile Total No of isolates

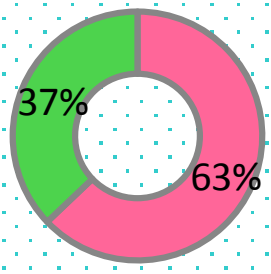


Diagram.2 Pie-diagram Showing Biofilm Production by Three Different Methods

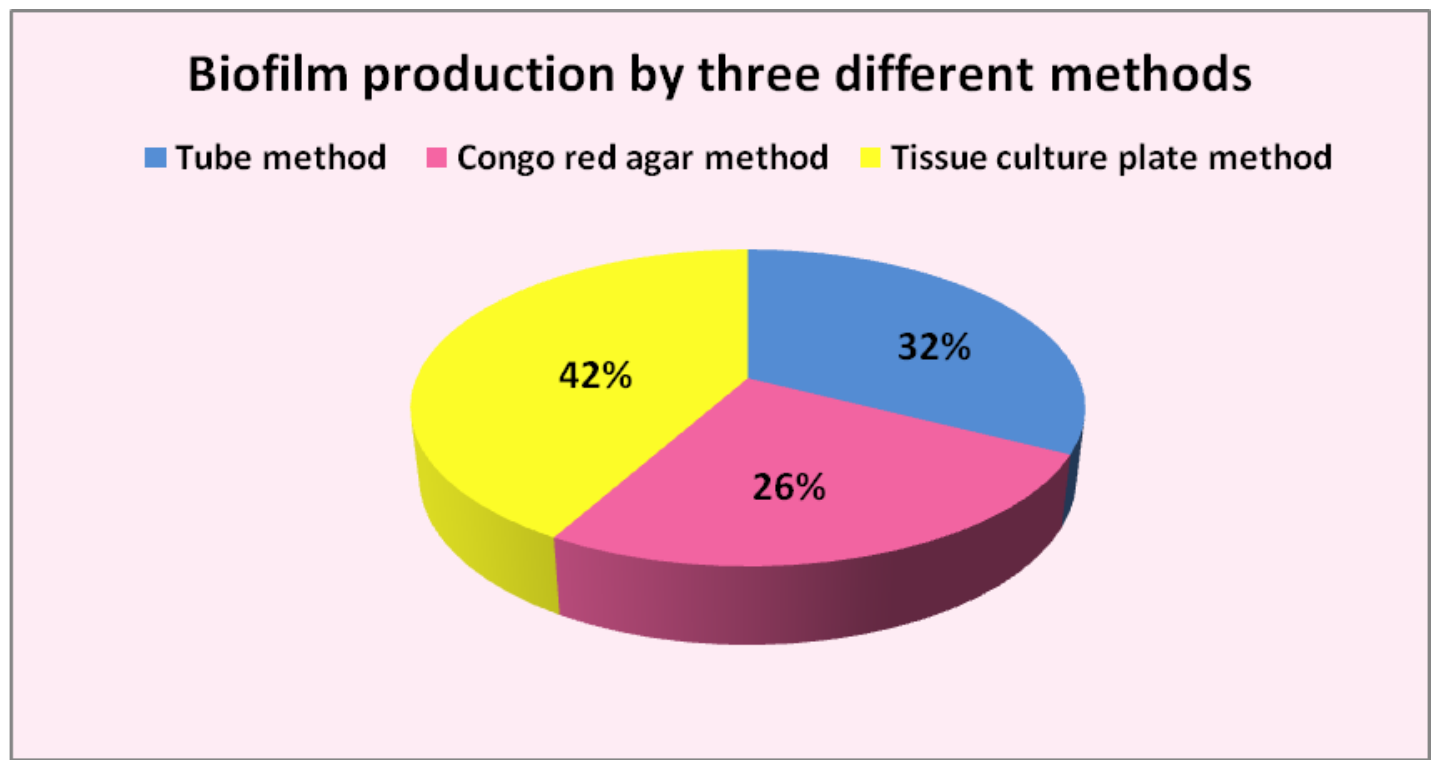

Diagram.3 Bar Diagram Showing the Isolates Forming Biofilms

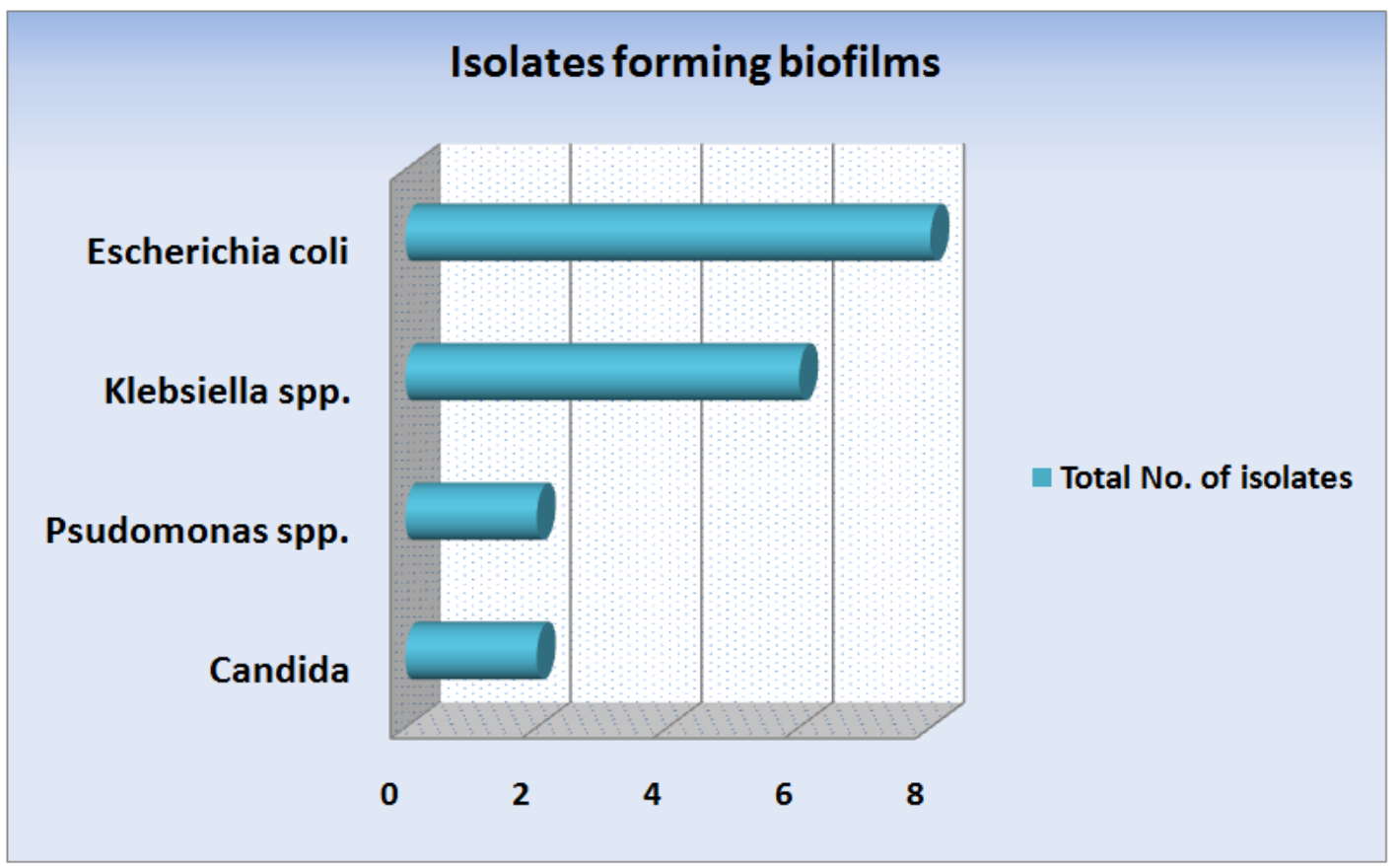


Diagram.4 Bar Diagram Showing the Co-relation between the Duration of Catheterization and the Biofilm Production

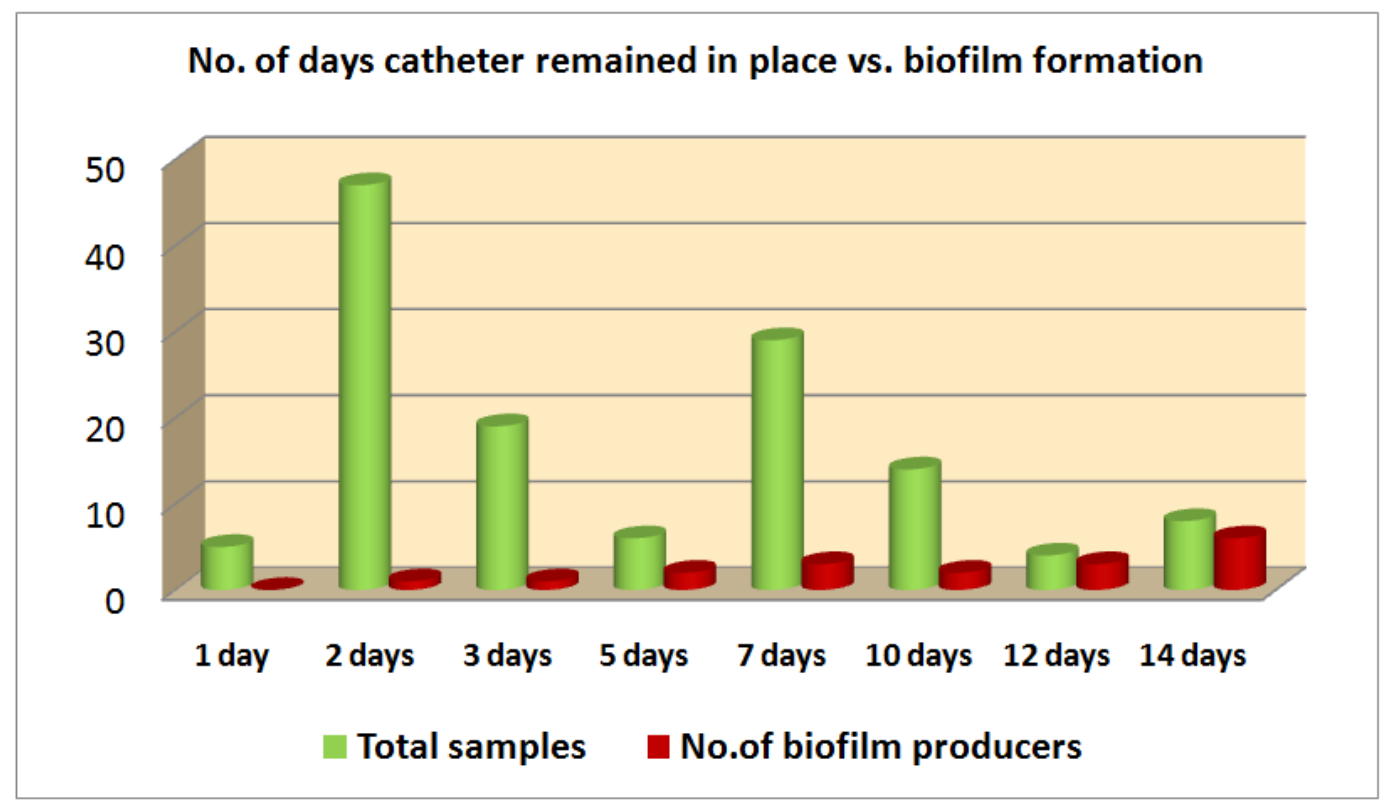

In the present study, $1 \%$ glucose was added to trypicase soy broth in TCP method and tube method and $10 \%$ sucrose to agar in CRA method. Thus, addition of sugar helps in biofilm formation. Similar findings were reported by Mathur T, et al., 2006 and Bose S, et al., (2009). In the present study, isolates showing biofilm production were Escherichia coli, Klebsiella spp. and Pseudomonas spp. These findings are consistent with that of Stickler,et al., (1996) who isolated Escherichia coli, Klebsiella spp., Pseudomonas spp. along with Proteus and Enterococcus faecalis from the tips of urinary catheters.

In conclusion, Biofilm producing bacteria are responsible for many recalcitrant infections and are notoriously difficult to eradicate $^{[12]}$. The information on the capacity of a clinical isolate toproduce biofilm would help a clinician to evaluate the measure of its virulence and devise an appropriate treatment plan for the patient
Detection of biofilm formation can help prevent potentially fatal and persistent infections ${ }^{[10]}$. Tissue Culture Plate method can be recommended as a general screening method for the detection of biofilm producing bacteria in laboratories ${ }^{[2]}$.

Wise usage of indwelling urinary catheters in patients and their timely replacement, preferably every two to three days; help prevent the formation of biofilms. For all post-operative cases, the catheter has to be removed as soon as possible, preferably within 24 hours to prevent biofilm formation [17].

\section{References}

1. Anuradha De, Dhanashree Deshpande, Sujata M Baveja, Shripad Taklikar. Detection of biofilm formation in bacteria from cases of urinary tract infections, septicemia, skin and soft tissue infections and post-operative infections by Congo Red Agar method. J Acad Med Sci 2012;2: 46-7. 
2. Hassan A, Usman J, Kaleem F, Omair M, Khalid A, Iqbal M. Evaluation of different detection methods for biofilm formation in the clinical isolates. Braz $\mathbf{J}$ Infect Dis 2011;15: 305-11.

3. Mathur T, Singhal S, Khan S, Upadhyay DJ, Fatma T, Rattan A. Detection of biofilm formation among the clinical isolates of Staphylococci: an evaluation of three different screening methods. Indian J Med Microbiol. 2006 Jan;24(1):25-9.

4. N.C. Maldonado, C. Silva de Ruiz, M. Cecilia and M.E.Nader-Macias. A simple technique to detect Klebsiella biofilmforming-strains. Inhibitory potential of Lactobacillus fermentum CRL 1058 whole cells and products. Communicating Current Research and Educational Topics and Trends in Applied Microbiology 2007: 5259.

5. Ryad Djeribi, WardaBouchloukh, Thierry Jouenne, Bouzid Menaa. Characterization of bacterial biofilms formed on urinary catheters.

6. Thaís Dias Lemos Kaiser, Eliezer Menezes Pereira, Kátia Regina Netto dos Santos, Ethel Leonor NoiaMaciel, Ricardo Pinto Schuenck, Ana Paula Ferreira Nunes. Modification of the Congo red agar method to detect biofilm production by Staphylococcus epidermidis. J Diag Microbio. Nov 2012.

7. Johannes K. M. Knobloch, Matthias A. Horstkotte, Holger Rohde, Dietrich Mack Evaluation of different detection methods of biofilm formation in Staphylococcus aureus. Medical Microbiology and Immunology October 2002, Volume 191, Issue 2, pp 101106.
8. Nabajit Deka. Comparison of Tissue Culture plate method, Tube Method and Congo Red Agar Method for the detection of biofilm formation by Coagulase Negative Staphylococcus isolated from Non-clinical Isolates. Int.J.Curr.Microbiol.App.Sci(2014) 3(10) 810-815.

9. Vasanthi R, Karthikeyan D, Jeya M. Study of biofilm production and antimicrobial resistance pattern of the bacterial isolates from invasive devices. Int J Res Health Sci [Internet]. 2014 Jan31;2(1):274-81.

10. YasmeenTaj, FarhanEssa, Faisal Aziz, Shahana U. Kazmi.Study on biofilmforming properties of clinical isolates of Staphylococcus aureus. J Infect DevCtries2012; 5(6):403-409.

11. Samant Sharvari A and Pai Chitra G. Evaluation of different detection methods of biofilmformation in clinical isolates of Staphylococci Int J Pharm Bio Sci 2012 Oct; 3(4): (b) $724-733$.

12. A.R. Rewatkar, Dr. B. J. Wadher Staphylococcus aureus and Pseudomonas aeruginosa Biofilm formation Methods. IOSR Journal of Pharmacy and Biological Sciences. Volume 8, Issue 5 (Nov. - Dec. 2013), PP 36-40.

13. Rise of Antimicrobial Resistance in ICU. The Critical Opinion July- Sep 2015:Issue 6.

14. Bailey and Scott's Diagnostic Microbiology, $13^{\text {th }}$ edition by Patricia M. Tille. Elsevier publications 2014: 35 .

15. Bose S, Khodke M, Basak S, Mallick SK. Detection of biofilm producing staphylococci: need of the hour. J Clin Diagn Res 2009;3: 1915-20.

16. Stickler. 1996. Bacterial biofilms and the encrustations of urethral catheter. Biofouling, vol 9, no 4, pp.293-305.

\section{How to cite this article:}

Venkata Hemalatha Neeli, T. Parvathi and Perala Balamurali Krishna. 2016. Study of Biofilm Production and Anti-microbial susceptibility pattern of bacterial and fungal isolates from urinary catheters. Int.J.Curr.Microbiol.App.Sci. 5(2): 415-424.

doi: http://dx.doi.org/10.20546/ijcmas.2016.502.046 